K. Ishige

Nagoya Math. J.

Vol. 155 (1999), 1-26

\title{
ON THE BEHAVIOR OF THE SOLUTIONS OF DEGENERATE PARABOLIC EQUATIONS
}

\author{
KAZUHIRO ISHIGE
}

\begin{abstract}
In this paper we consider degenerate parabolic equations, and obtain an interior and a boundary Harnack inequalities for nonnegative solutions to the degenerate parabolic equations. Furthermore we obtain boundedness and continuity of the solutions.
\end{abstract}

\section{$\S 1$. Introduction}

We consider the degenerate parabolic equation

$$
\begin{aligned}
\frac{\partial}{\partial t} u=\frac{1}{w(x)} \sum_{i, j=1}^{N} \frac{\partial}{\partial x_{j}} & \left(a_{i j}(x, t) w(x) \frac{\partial}{\partial x_{i}} u\right) \\
& +\sum_{i=1}^{N} b_{i}(x, t) \frac{\partial}{\partial x_{i}} u-V(x, t) u
\end{aligned}
$$

in $D=\Omega \times(-1,1)$, where $\Omega$ is a domain in $\mathbf{R}^{N}$. Here $w$ is a nonnegative function in $\Omega$, and $a_{i j}, b_{i}$, and $V, i, j=1, \ldots, N$, are measurable functions defined on $D$. In this paper we study the behavior of solutions of the equation (1.1), and give an interior and a boundary Harnack inequalities for nonnegative solutions of (1.1).

An interior Harnack inequality for parabolic equations was first obtained by J. Moser [Ms], and was extended to more general parabolic equations by many authors (cf. [CS1,2,3], [GW1,2], [Mr], [Se], [Sta], [T] and references therein). F. Chiarenza and R. Serapioni [CS1,2,3] obtained the interior Harnack inequality of nonnegative solutions of degenerate parabolic equations of the types, $u_{t}=\mathcal{L}_{w} u$ and $u_{t}=w^{-1} \mathcal{L}_{w} u$, where

$$
\mathcal{L}_{w} u \equiv \sum_{i, j=1}^{N} \frac{\partial}{\partial x_{j}}\left(a_{i j}(x, t) w(x) \frac{\partial}{\partial x_{i}} u\right) .
$$

Received October 16, 1997. 
Subsequently, the results of $[\mathrm{CS} 1,2,3]$ were extended to more general degenerate parabolic equations (cf. [CW], [GW1,2], [SaC1,2], and references therein).

A boundary Harnack inequality for parabolic equations was first obtained by J. K. Kemper [K]. He obtained the boundary Harnack inequality for nonnegative solutions of the heat equation in Lipschitz domains. Furthermore, P. Salsa [Sa] extended the result of $[\mathrm{K}]$ to the parabolic equation $u_{t}=\mathcal{L}_{w} u$ in Lipschitz domains for the case that $w$ is constant (cf. [FGS]).

In this paper we extend the results of [CS3] and [Sa], and obtain the interior and the boundary Harnack inequalities for nonnegative solutions of the degenerate parabolic equation (1.1). Furthermore, we obtain boundedness and continuity of solutions of (1.1).

We introduce some notations. For any $(x, t) \in \mathbf{R}^{N+1}$ and $\rho>0$, set

$$
\begin{aligned}
B(x, \rho) & =\left\{y \in \mathbf{R}^{N}|| x-y \mid<\rho\right\}, \\
Q_{x, t}(\rho) & =B(x, 2 \rho) \times\left(t-\rho^{2}, t+\rho^{2}\right), \\
Q_{x, t}^{-}(\rho) & =B(x, \rho) \times\left(t-\frac{3}{4} \rho^{2}, t-\frac{1}{4} \rho^{2}\right), \\
Q_{x, t}^{+}(\rho) & =B(x, \rho) \times\left(t+\frac{1}{4} \rho^{2}, t+\frac{3}{4} \rho^{2}\right) .
\end{aligned}
$$

For simplicity, we put $Q(\rho)=Q_{0,0}(\rho), Q=Q_{0,0}(1)$, and $Q^{ \pm}=Q_{0,0}^{ \pm}(1)$. Furthermore, for any measurable set $E \subset \mathbf{R}^{N}$, we denote by $|E|$ the the Lebesgue measure of $E$. and $w$ :

We impose the following conditions on the coefficients $\left\{a_{i j}(x, t)\right\}_{i, j=1}^{N}$

(A1) There exists a constant $\lambda>0$ such that

$$
\lambda|\xi|^{2} \leq \sum_{i, j=1}^{N} a_{i j}(x, t) \xi_{i} \xi_{j} \leq \lambda^{-1}|\xi|^{2}, \quad \xi \in \mathbf{R}^{N},(x, t) \in D
$$

(A2) Let $w$ be an $A_{2}$ weight in $\Omega$, that is, $w, w^{-1} \in L_{\mathrm{loc}}^{1}(\Omega)$, and there exists a constant $c_{0}$ such that

$$
\sup _{\substack{E \subset \Omega \\ E \text { is a cube }}} \frac{1}{|E|^{2}} \int_{E} w d x \int_{E} \frac{d x}{w} \leq c_{0} .
$$


We denote by $L^{p}(\Omega, w d x), 1 \leq p<\infty$, the Banach space of all measurable functions $f$ defined on $\Omega$ such that

$$
\|f\|_{L^{p}(\Omega, w d x)} \equiv\left(\int_{\Omega}|f(x)|^{p} w(x) d x\right)^{\frac{1}{p}}<\infty .
$$

Furthermore, we denote by $H_{0}^{1}(\Omega, w d x)$ and $H^{1}(\Omega, w d x)$ the closure of $C_{0}^{\infty}(\Omega)$ and $C^{\infty}(\bar{\Omega})$ under the norm

$$
\left(\int_{\Omega}|f(x)|^{2} w(x) d x+\int_{\Omega}|\nabla f(x)|^{2} w(x) d x\right)^{\frac{1}{2}}
$$

respectively. For measurable sets $E \subset \Omega, F \subset D$ and $f \in L_{\mathrm{loc}}^{1}(\Omega, w d x)$, set

$$
\begin{aligned}
& w(E)=\int_{E} w(x) d x, \quad(w \otimes 1)(F)=\iint_{F} w(x) d x d \tau \\
& f_{E} f w d x=\frac{1}{w(E)} \int_{E} f w d x
\end{aligned}
$$

We recall the Sobolev inequality with weight: There exist constants $c_{1}>0$ and $\kappa>1$ depending only on $c_{0}$ such that

$$
\left(f_{B(x, \rho)}|u|^{2 \kappa} w(y) d y\right)^{\frac{1}{2 \kappa}} \leq c_{1} \rho\left(f_{B(x, \rho)}|\nabla u|^{2} w(y) d y\right)^{\frac{1}{2}}
$$

for all functions $u \in H_{0}^{1}(B(x, \rho), w d x)$ and all $B(x, \rho) \subset \Omega$. It is known that $\kappa>\frac{N}{N-1}$ for $N \geq 2$ and $\kappa$ is any number for $N=1$. (cf. See [FKS].) Furthermore, for the case that $w \equiv 1$ on $\Omega, \kappa=\frac{N}{N-2}$ if $N \geq 3$ and $\kappa$ is any number for $N=1,2$. For further details on weight functions, see [HKO] and [Ste].

Throughout this paper, we assume that there exists a positive constant $\epsilon$ such that

$$
\begin{aligned}
& b \in L^{\infty}\left((-1,1): L^{2 \kappa^{\prime}}(\Omega, w d x)\right), \\
& V \in L^{\infty}\left((-1,1): L^{\kappa^{\prime}+\epsilon}(\Omega, w d x)\right),
\end{aligned}
$$

where $b=\left(b_{1}, \ldots, b_{N}\right)$ and $\kappa^{\prime}$ is a constant with $\frac{1}{\kappa}+\frac{1}{\kappa^{\prime}}=1$. We say that $u$ is a solution of (1.1) in $D$ when $u$ is a measurable function in $D$ belonging 
to $L^{\infty}\left((-1,1): L^{2}(\Omega, w d x)\right) \cap L^{2}\left((-1,1): H^{1}(\Omega, w d x)\right)$ and satisfies

$$
\begin{aligned}
\iint_{D}\left[-u \frac{\partial \varphi}{\partial \tau}\right. & +\sum_{i, j=1}^{N} a_{i j}(x, \tau) \frac{\partial u}{\partial x_{i}} \frac{\partial \varphi}{\partial x_{j}} \\
& \left.-\sum_{i=1}^{N} b_{i}(x, \tau) \frac{\partial u}{\partial x_{i}} \varphi+V(x, \tau) u \varphi\right] w(x) d x d \tau=0
\end{aligned}
$$

for any $\varphi \in C_{0}^{\infty}(D)$.

In order to state our results, we furthermore impose some conditions on $b$. In this Introduction, for simplicity, we assume the following condition, and state our results.

(A4) There exist measurable functions $c$ and $d$ defined on $D$ such that

(i) $\quad b(x, t)=c(x, t)+d(x, t), \quad$ for almost all $(x, t) \in D$,

(ii) $\quad c \in C\left([-1,1]: L^{2 \kappa^{\prime}}(\Omega, w d x)\right), \quad d \in L^{\infty}\left((-1,1): L^{2\left(\kappa^{\prime}+\epsilon\right)}(\Omega, w d x)\right)$.

Theorem A. Assume (A1)-(A4). Let $Q \subset D$ and $u$ be a nonnegative solution of (1.1) in $Q$. Then there exists a constant $C$ such that

$$
\sup _{Q^{-}} u \leq C \inf _{Q^{+}} u
$$

ThEOREM B. Assume (A1)-(A4) and the following condition:

(A5) If $|b|+|V| \not \equiv 0$ on $D$, there exists a positive constant $c_{2}$ such that

$$
c_{2} \rho^{2 \kappa^{\prime}} \leq w(B(x, \rho)), \quad \rho \in(0,1), B(x, \rho) \subset \Omega .
$$

Let $u$ be a solution of (1.1) in D. Then $u$ is a locally Hölder continuous function in D. Furthermore, there exist positive constants $C$ and $\delta$ such that

$$
\underset{Q_{x, t}(\rho)}{\operatorname{Osc}} u \leq C\left(\frac{\rho}{\rho_{1}}\right)^{\delta}\left(\underset{Q_{x, t}\left(\rho_{1}\right)}{\operatorname{osc}} u+\rho_{1}^{\frac{2 \epsilon}{\kappa^{\prime}+\epsilon}}\|u\|_{L^{\infty}\left(Q_{x, t}\left(\rho_{1}\right)\right)}\right)
$$

for all $0<\rho<\rho_{1} \leq \frac{1}{2}$ with $Q_{x, t}\left(\rho_{1}\right) \subset D$. Here $\underset{Q_{x, t}(\rho)}{\operatorname{osc}} u=\sup _{Q_{x, t}(\rho)} u-$ $\inf _{Q_{x, t}(\rho)} u$.

TheOrem C. Let $x_{0} \in \partial \Omega$. Assume that there exist a positive constant $r_{0}$ and an orthonormal system such that $\Omega \cap B\left(x_{0}, r_{0}\right)$ is described as follows:

$$
\begin{aligned}
\Omega \cap B\left(x_{0}, r_{0}\right) & =\left\{\left(x^{\prime}, x_{n}\right) \mid x^{\prime} \in \mathbf{R}^{N-1}, x_{n}>\varphi\left(x^{\prime}\right)\right\} \cap B\left(x_{0}, r_{0}\right), \\
\partial \Omega \cap B\left(x_{0}, r_{0}\right) & =\left\{\left(x^{\prime}, \varphi\left(x^{\prime}\right)\right) \mid x^{\prime} \in \mathbf{R}^{N-1}\right\} \cap B\left(x_{0}, r_{0}\right),
\end{aligned}
$$


where $\varphi(\cdot)$ is a Lipschitz continuous function on $\mathbf{R}^{N-1}$ with Lipschitz constant $m$. Assume (A1)-(A5). Let $u$ be a nonnegative solution of (1.1) in $D$ vanishing continuously on $\left[\partial \Omega \cap B\left(x_{0}, r_{0}\right)\right] \times(-1,1)$. Then there exists a positive constant $C$ such that

$$
\begin{aligned}
& u(x, t) \leq C u\left(\left(x_{0}^{\prime}, \varphi\left(x_{0}^{\prime}\right)+r\right), s+2 r^{2}\right), \\
& s \in(-1,1), r<\frac{1}{4} \min \left\{r_{0}, 2 \sqrt{1-|s|},\right.
\end{aligned}
$$

for $(x, t) \in D \cap\left\{(x, t) \in \mathbf{R}^{N+1}|| x-x_{0}|<r| t-s \mid,<r^{2}\right\}$.

We remark that the condition (A3) is a necessary one for Theorems $\mathrm{A}, \mathrm{B}$, and $\mathrm{C}$ to hold. Let $N \geq 3$, and set $u_{1}(x)=|x|^{-\frac{1}{4}}$ and $u_{2}(x)=$ $-\log |x|$. Then $u_{i} \in L^{2}(B(0,1)) \cap H^{1}(B(0,1))$ and $u_{i} \notin L^{\infty}(B(0,1)), i=$ 1,2. Furthermore, $u_{1}$ and $u_{2}$ satisfy the elliptic equations,

$$
\begin{array}{lll}
\Delta u-\frac{(4 N-9) x}{4|x|^{2}} \nabla u=0 & \text { in } \quad B(0,1), & \frac{x}{|x|^{2}} \notin L^{N}(B(0,1)), \\
\Delta u-\frac{N-2}{|x|^{2} \log |x|} u=0 & \text { in } \quad B(0,1), & \frac{1}{|x|^{2} \log |x|} \in L^{\frac{N}{2}}(B(0,1)),
\end{array}
$$

respectively. Therefore, for the equation

$$
\begin{aligned}
u_{t}=\Delta u+b(x) \nabla u-V(x) u & \text { in } \quad B(0,1) \times(-1,1), \\
b & \in L^{p}(B(0,1)), \quad V \in L^{q}(B(0,1)),
\end{aligned}
$$

if $p<N$ or $q=\frac{N}{2}$, the results of the theorems don't necessary hold. If $p \geq N$ and $q>\frac{N}{2}$, the equation (1.9) satisfies the conditions (A3), and the results of the theorems hold.

We modify the arguments of [CS3], [LSU], [Ms], [Sa], [Se], and [Sta], and prove our theorems. However, for the case $b \in\left[L^{\infty}\left((-1,1): L^{2 \kappa^{\prime}}(\Omega)\right) \backslash\right.$ $\left.L^{\infty}\left((-1,1): L^{p}(\Omega)\right)\right], p>2 \kappa^{\prime}$, it seems difficult to apply their arguments directly. The main difficulty is to obtain $L^{\infty}$-estimates of solutions of the equation (1.1). To overcome this difficulty, we first prove the boundedness of solutions by using De Giorgi's iteration method. Next we obtain an $L^{\infty}$ estimate of solutions by using boundedness of solutions and De Giorgi's iteration method.

The rest of this paper is organized as follows. In Section 2 we obtain an $L^{\infty}$-estimate of nonnegative solutions of (1.1). In Section 3 we use the $L^{\infty}$-estimate of nonnegative solutions obtained in Section 2 , and prove the 
interior Harnack inequality. Furthermore, we obtain some inequalities of solutions by using the interior Harnack inequality, and prove the continuity of solutions. In Section 4 we use the results of Section 3, and prove the boundary Harnack inequality.

\section{$\S 2 . \quad L_{\text {loc }}^{\infty}$ estimates of solutions}

In this section, by using De Giorgi's iteration method, we obtain $L^{\infty}$ estimates of solutions of the degenerate parabolic equation (1.1).

Throughout this section, we will prove the following proposition.

Proposition 2.1. Let $Q \subset D$. Assume (A1)-(A3), and the following condition:

(A4.a) There exist a constant $\sigma$ and measurable functions $c$ and d defined on $Q$ such that

(i) $b(x, t)=c(x, t)+d(x, t), \quad$ for almost all $(x, t) \in Q$,

(ii) $\sup _{-1<t<1} f_{B(0,2)}|c(x, t)|^{2 \kappa^{\prime}} w(x) d x \leq \sigma$,

(iii) $d \in L^{\infty}\left((-1,1): L^{2\left(\kappa^{\prime}+\epsilon\right)}(B(0,2), w d x)\right)$.

Let $u$ be a nonnegative solution of (1.1) in $Q$. Then there exists a constant $\sigma_{1}=\sigma_{1}\left(N, c_{0}, \epsilon\right)$ such that, if $\sigma \leq \sigma_{1}, u$ is an $L_{\mathrm{loc}}^{\infty}(Q)$ function satisfying

$$
\|u\|_{L_{\mathrm{loc}}^{\infty}(Q(\rho))} \leq\left(\frac{C\left(\rho^{\prime}-\rho\right)^{-\mu}}{(w \otimes 1)\left(Q\left(\rho^{\prime}\right)\right)} \iint_{Q\left(\rho^{\prime}\right)} u^{p} w d x d \tau\right)^{\frac{1}{p}},
$$

for all $\frac{1}{2} \leq \rho<\rho^{\prime} \leq 1$ and $0<p \leq 1+\frac{\kappa^{\prime}}{\epsilon}$. Here $C$ is a constant depending only on $N, \lambda, c_{0}, \epsilon$, and

$$
\mathcal{D} \equiv \sup _{-1<t<1} f_{B(0,2)}\left[|d(x, t)|^{2}+|V(x, t)|\right]^{\kappa^{\prime}+\epsilon} w(x) d x .
$$

In order to prove Proposition 2.1, we recall the following lemma (see [CS3]).

Lemma 2.2. Assume $(\mathrm{A} 2)$ and $B\left(x_{0}, \rho\right) \subset \Omega$. Then

$$
\left(\frac{1}{b-a} \int_{a}^{b} f_{B\left(x_{0}, \rho\right)}|u|^{2 \bar{\kappa}} w d x d \tau\right)^{\frac{1}{2 \bar{\kappa}}}
$$




$$
\begin{aligned}
\leq\left(c_{1} \rho\right)^{\frac{1}{\bar{\kappa}}}\left(\sup _{a<\tau<b} f_{B\left(x_{0}, \rho\right)} u^{2} w d x\right)^{\frac{1}{2}\left(1-\frac{1}{\bar{\kappa}}\right)} \\
\quad \times\left(\frac{1}{b-a} \int_{a}^{b} f_{B\left(x_{0}, \rho\right)}|\nabla u|^{2} w d x d \tau\right)^{\frac{1}{2 \bar{\kappa}}}
\end{aligned}
$$

for $u \in L^{\infty}\left((a, b): L^{2}\left(B\left(x_{0}, \rho\right), w d x\right)\right) \cap L^{2}\left((a, b): H_{0}^{1}\left(B\left(x_{0}, \rho\right), w d x\right)\right)$, where $\bar{\kappa}=\frac{2 \kappa-1}{\kappa}>1$ and $c_{1}$ is a constant appearing in (1.3).

By using Lemma 2.2, we prove the following two lemmas, in which we prove boundedness of nonnegative solutions of (1.1).

Lemma 2.3. Assume the same conditions as those of Proposition 2.1. Let $u$ be a nonnegative subsolution of (1.1) in $Q$. Then there exists a constant $p=p\left(c_{0}, \epsilon\right)$ such that if $u$ belongs to $L_{\mathrm{loc}}^{p}(Q, w d x d \tau)$, then $u$ is a function in $L_{\mathrm{loc}}^{\infty}(Q)$.

Proof of Lemma 2.3. Let $\frac{1}{2} \leq \rho<\rho^{\prime} \leq 1$, and consider a sequence $\rho_{n}=\rho+2^{-n}\left(\rho^{\prime}-\rho\right), n=0,1,2, \ldots$ We denote by $\zeta_{n}$ a nonnegative piecewise smooth function in $Q\left(\rho_{n}\right)$ such that $\zeta_{n}=1$ on $Q\left(\rho_{n+1}\right), \operatorname{supp} \zeta_{n} \subset Q\left(\rho_{n}\right)$, and

$$
\left|\nabla \zeta_{n}\right|^{2}+\left|\left(\zeta_{n}\right)_{\tau}\right| \leq 2^{2(n+1)} /\left(\rho^{\prime}-\rho\right)^{2} .
$$

Let $f_{+}=\max (f, 0)$ for any measurable function $f$ in $D$. For any $k>0$, put $u_{k}=(u-k)_{+}$and $k_{n}=k\left(1-2^{-(n+1)}\right)$.

Let $p$ be a constant to be chosen later such that $p \geq 1+\frac{\kappa^{\prime}}{\epsilon}$. We multiply the equation (1.1) by $u_{k_{n+1}}^{r} \zeta_{n}^{2}, r=\frac{\kappa^{\prime}}{\epsilon}$. Then we obtain by standard calculations (see [LSU, Chapter 3, §2])

$$
\begin{gathered}
\frac{1}{1+r} \sup _{-\rho_{n}^{2}<\tau<\rho_{n}^{2}} \int_{B\left(0, \rho_{n}\right)} u_{k_{n+1}}^{r+1} \zeta_{n}^{2} w d x+\frac{r}{(r+1)^{2}} \\
\times \iint_{Q\left(\rho_{n}\right)}\left|\nabla u_{k_{n+1} \frac{r+1}{2}}^{2}\right|^{2} \zeta_{n}^{2} w d x d \tau \\
\leq C_{1}\left(1+r^{-1}\right) \int_{Q\left(\rho_{n}\right)}\left[\left(|c(x, \tau)|^{2}+|d(x, \tau)|^{2}\right) u_{k_{n+1}+1}^{r+1}\right. \\
\left.+|V(x, \tau)| u u_{k_{n+1}}^{r}\right] \zeta_{n}^{2} w d x d \tau \\
+C_{1}\left(1+r^{-1}+(1+r)^{-1}\right) \\
\times \iint_{Q\left(\rho_{n}\right)} u_{k_{n+1}^{r+1}\left[\left|\nabla \zeta_{n}\right|^{2}+\zeta_{n}\left|\left(\zeta_{n}\right)_{\tau}\right|\right] w d x d \tau}
\end{gathered}
$$


for some constant $C_{1}=C_{1}\left(\lambda, N, c_{0}\right)$. On the other hand, by the doubling property of the $A_{2}$ weight $w$, there exists a constant $c_{3}$ depending only on $c_{0}$ such that

$$
w(B(0,2)) \leq c_{3} w\left(B\left(0,2 \rho_{n}\right)\right), \quad n=0,1, \ldots
$$

By (1.3) and (2.5),

(2.6) $\iint_{Q\left(\rho_{n}\right)}|c(x, \tau)|^{2} u_{k_{n+1}}^{r+1} \zeta_{n}^{2} w d x d \tau$

$$
\begin{aligned}
& \leq \sup _{-\rho_{n}^{2}<\tau<\rho_{n}^{2}}\left(\int_{B\left(0,2 \rho_{n}\right)}|c(x, \tau)|^{2 \kappa^{\prime}} w d x\right)^{\frac{1}{\kappa^{\prime}}} \\
& \quad \times \int_{-\rho_{n}^{2}}^{\rho_{n}^{2}}\left(\int_{B\left(0,2 \rho_{n}\right)}\left(u_{k_{n+1}}^{\frac{r+1}{2}} \zeta_{n}\right)^{2 \kappa} w d x\right)^{\frac{1}{\kappa}} d \tau \\
& \leq 4 c_{1}^{2} \rho_{n}^{2}\left(\frac{\sigma_{1} w(B(0,2))}{w\left(B\left(0,2 \rho_{n}\right)\right)}\right)^{\frac{1}{\kappa^{\prime}}} \iint_{Q\left(\rho_{n}\right)}\left|\nabla\left[u_{k_{n+1}}^{\frac{r+1}{2}} \zeta_{n}\right]\right|^{2} w d x d \tau \\
& \leq 4 c_{1}^{2}\left(\sigma_{1} c_{3}\right)^{\frac{1}{\kappa^{\prime}}} \iint_{Q\left(\rho_{n}\right)}\left|\nabla\left[u_{k_{n+1}}^{\frac{r+1}{2}} \zeta_{n}\right]\right|^{2} w d x d \tau .
\end{aligned}
$$

If $\sigma_{1}$ is a sufficiently small constant such that

$$
4 c_{1}^{2} C_{1}\left(1+r^{-1}\right)\left(\sigma_{1} c_{3}\right)^{\frac{1}{\kappa^{\prime}}}<\frac{r}{2(r+1)^{2}},
$$

then there exists a constant $C_{2}>0$ such that

$$
\begin{array}{r}
(2.7) \sup _{-\rho_{n}^{2}<\tau<\rho_{n}^{2}} \int_{B\left(0,2 \rho_{n}\right)} u_{k_{n+1}}^{r+1} \zeta_{n}^{2} w d x+\iint_{Q\left(\rho_{n}\right)}\left|\nabla u_{k_{n+1}}^{\frac{r+1}{2}}\right|^{2} \zeta_{n}^{2} w d x d \tau \\
\leq C_{2} \iint_{Q\left(\rho_{n}\right)}\left[|d(x, \tau)|^{2} u_{k_{n+1}}^{r+1} \zeta_{n}^{2}+|V(x, \tau)| u u_{k_{n+1}}^{r} \zeta_{n}^{2}\right] w d x d \tau \\
+C_{2} \iint_{Q\left(\rho_{n}\right)} u_{k_{n+1}}^{r+1}\left[\left|\nabla \zeta_{n}\right|^{2}+\zeta_{n}\left|\left(\zeta_{n}\right)_{\tau}\right|\right] w d x d \tau
\end{array}
$$

By the Hölder inequality and the similar way to (2.6), for any $\nu>0$, there exist constants $C_{3}(\nu)$ and $C_{4}\left(c_{0}, \mathcal{D}\right)$ such that

(2.8) $\iint_{Q\left(\rho_{n}\right)}\left[|d(x, \tau)|^{2} u_{k_{n+1}}^{r+1}+|V(x, \tau)| u u_{k_{n+1}}^{r}\right] \zeta_{n}^{2} w d x d \tau$ 


$$
\begin{aligned}
& \leq \nu \iint_{Q\left(\rho_{n}\right)}\left[|d|^{2}+|V|\right]^{\frac{\kappa^{\prime}+\epsilon}{\kappa^{\prime}}} u_{k_{n+1}}^{r+1} \zeta_{n}^{2} w d x d \tau \\
& \quad+C_{3} \iint_{Q\left(\rho_{n}\right)} u^{r+1} \chi_{\left\{u>k_{n+1}\right\}} \zeta_{n}^{2} w d x d \tau \\
& \leq \nu C_{4} \iint_{Q\left(\rho_{n}\right)}\left|\nabla\left[u_{k_{n+1}^{\frac{r+1}{2}}} \zeta_{n}\right]\right|^{2} w d x d \tau \\
& +C_{3} \iint_{Q\left(\rho_{n}\right)} u^{r+1} \chi_{\left\{u>k_{n+1}\right\}} \zeta_{n}^{2} w d x d \tau .
\end{aligned}
$$

We take a sufficiently small $\nu>0$ such that $\nu C_{2} C_{4}<\frac{1}{2}$. By $(2.7)-(2.8)$,

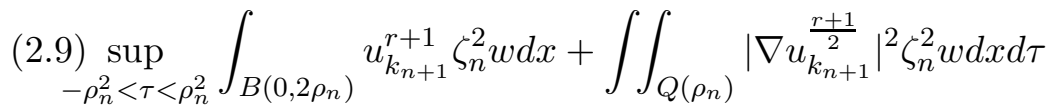

$$
\begin{aligned}
& \leq C_{5} \iint_{Q\left(\rho_{n}\right)} u^{r+1} \chi_{\left\{u>k_{n+1}\right\}}\left[\zeta_{n}^{2}+\left|\nabla \zeta_{n}\right|^{2}+\zeta_{n}\left|\left(\zeta_{n}\right)_{\tau}\right|\right] w d x d \tau
\end{aligned}
$$

for some constant $C_{5}>0$. Let $p=q(r+1)$, where $q$ is a constant to be chosen later such that $q>1$. Then, by the Hölder inequality

$$
\begin{aligned}
& \iint_{Q\left(\rho_{n}\right)} u^{r+1} \chi_{\left\{u>k_{n+1}\right\}} w d x d \tau \\
& \leq\left(\iint_{Q\left(\rho_{n}\right)} u^{q(r+1)} w d x d \tau\right)^{\frac{1}{q}}\left(\iint_{Q\left(\rho_{n}\right)} \chi_{\left\{u>k_{n+1}\right\}} w d x\right)^{1-\frac{1}{q}} \\
& \leq\left(\iint_{Q\left(\rho_{n}\right)} u^{q(r+1)} w d x d \tau\right)^{\frac{1}{q}}\left(\left|k_{n+1}-k_{n}\right|^{-(r+1)} \iint_{Q\left(\rho_{n}\right)} u_{k_{n}}^{r+1} w d x d \tau\right)^{1-\frac{1}{q}}, \\
& \leq\left(\iint_{Q\left(\rho_{n}\right)} u^{q(r+1)} w d x d \tau\right)^{\frac{1}{q}}\left(\frac{2^{(r+1)(n+1)}}{k^{r+1}} \iint_{Q\left(\rho_{n}\right)} u_{k_{n}}^{r+1} w d x d \tau\right)^{1-\frac{1}{q}} .
\end{aligned}
$$

By Lemma 2.2, there exists a constant $C_{6}$ such that

$$
\begin{aligned}
& \iint_{Q\left(\rho_{n+1}\right)} u_{k_{n+1}}^{(r+1) \bar{\kappa}} w d x d \tau \\
& \leq C_{6} \iint_{Q\left(\rho_{n}\right)}\left|\nabla\left[u_{k_{n+1}}^{\frac{r+1}{2}} \zeta_{n}\right]\right|^{2} w d x d \tau \\
& \quad \times\left(\frac{1}{w\left(B\left(0,2 \rho_{n}\right)\right)} \sup _{-\rho_{n}^{2} \leq \tau<\rho_{n}^{2}} \int_{B\left(0,2 \rho_{n}\right)} u_{k_{n+1}^{r+1}} \zeta_{n}^{2} w d x\right)^{\bar{\kappa}-1}
\end{aligned}
$$


Set

$$
y_{n}=\frac{1}{w\left(B\left(0,2 \rho_{n}\right)\right)} \iint_{Q\left(\rho_{n}\right)} u_{k_{n}}^{r+1} w d x d \tau
$$

By the Hölder inequality, (2.3) and (2.9)-(2.11), there exists a constant $C_{7}$ such that

$$
\begin{aligned}
& y_{n+1} \leq\left(\frac{1}{w\left(B\left(0,2 \rho_{n+1}\right)\right)} \iint_{Q\left(\rho_{n+1}\right)} u_{k_{n+1}}^{(r+1) \bar{\kappa}} w d x d \tau\right)^{\frac{1}{\bar{\kappa}}} \\
& \times\left(\frac{1}{w\left(B\left(0,2 \rho_{n+1}\right)\right)} \iint_{Q\left(\rho_{n+1}\right)} \chi_{\left\{u>k_{n+1}\right\}} w d x d \tau\right)^{1-\frac{1}{\bar{\kappa}}} \\
& \leq C_{7} 2^{2(n+1)} K\left(\frac{2^{(r+1)(n+1)}}{k^{r+1}} y_{n}\right)^{1-\frac{1}{q}}\left(\frac{2^{(r+1)(n+1)}}{k^{r+1}} y_{n}\right)^{1-\frac{1}{\bar{\kappa}}} \\
& \leq C_{7} K 2^{b_{n}} k^{-(r+1)\left(1+\mu_{1}\right)} y_{n}^{1+\mu_{1}}
\end{aligned}
$$

where $b_{n}=2(n+1)+2(n+1)(r+1)\left(1+\mu_{1}\right), \mu_{1}=1-\frac{1}{q}-\frac{1}{\bar{\kappa}}$, and

$$
K=\left(\frac{1}{w\left(B\left(0,2 \rho^{\prime}\right)\right)} \iint_{Q\left(\rho^{\prime}\right)} u^{q(r+1)} w d x d \tau\right)^{\frac{1}{q}}\left(\rho^{\prime}-\rho\right)^{-2} .
$$

Let $q$ be a constant such that $\mu_{1}>0$. By Lemma 5-6 of Chapter II in [LSU], there exists a constant $C_{8}>0$ such that if

$$
y_{0} \leq C_{8} k^{\frac{(r+1)\left(1+\mu_{1}\right)}{\mu_{1}}} K^{-\frac{1}{\mu_{1}}}
$$

then $\lim _{n \rightarrow \infty} y_{n}=0$. So we have

$$
\|u\|_{L^{\infty}(Q(\rho))} \leq\left(C_{8}^{-1} K^{\frac{1}{\mu_{1}}} \frac{1}{w\left(B\left(0,2 \rho^{\prime}\right)\right)} \iint_{Q\left(\rho^{\prime}\right)} u^{r+1} w d x d \tau\right)^{\frac{\mu_{1}}{(r+1)\left(1+\mu_{1}\right)}}
$$

By the arbitrariness of $\rho$ and $\rho^{\prime}$, if $u \in L_{\mathrm{loc}}^{q(r+1)}(Q, w d x d \tau)$, then $u \in L_{\mathrm{loc}}^{\infty}(Q)$. Therefore the proof of Lemma 2.3 is complete.

Lemma 2.4. Assume the same conditions as those of Proposition 2.1. Let $u$ be a nonnegative subsolution of (1.1) in $Q$. Then $u$ is a function in $L_{\mathrm{loc}}^{\infty}(Q)$. 
Proof of Lemma 2.4. Let $\frac{1}{2} \leq \rho<\rho^{\prime} \leq 1$. Let $\zeta$ be a piecewise smooth function in $Q\left(\rho^{\prime}\right)$ such that $0 \leq \zeta \leq 1, \zeta(x, t)=1$ on $Q(\rho), \operatorname{supp} \zeta \subset Q\left(\rho^{\prime}\right)$, and

$$
|\nabla \zeta|^{2}+\left|\zeta_{\tau}\right| \leq 4\left(\rho^{\prime}-\rho\right)^{-2}
$$

For $l>0$, set $u_{l}=\min \{u, l\}$. Let $\delta>0$. We multiply the equation (1.1) by $u\left(u_{l}+\delta\right)^{r} \zeta^{2}, r>0$. By calculating it in the similar way to (2.4) and letting $\delta \rightarrow 0$, we have

$$
\begin{aligned}
\sup _{-\left(\rho^{\prime}\right)^{2}<\tau<\left(\rho^{\prime}\right)^{2}} \int_{B\left(0,2 \rho^{\prime}\right)} & \frac{u_{l}^{r+2}}{r+2} \zeta^{2} w d x \\
& +\iint_{Q\left(\rho^{\prime}\right)}\left[u_{l}^{r}|\nabla u|^{2}+r u_{l}^{r}\left|\nabla u_{l}\right|^{2}\right] \zeta^{2} w d x d \tau \\
\leq C_{1}\left(1+r^{-1}\right) & \iint_{Q\left(\rho^{\prime}\right)}\left[|c(x, \tau)|^{2}+|d(x, \tau)|^{2}\right. \\
& +|V(x, \tau)|] u^{2} u_{l}^{r} \zeta^{2} w d x d \tau \\
& +C_{1}\left(1+r^{-1}+(r+2)^{-1}\right) \\
& \times \iint_{Q\left(\rho^{\prime}\right)} u^{2} u_{l}^{r}\left[|\nabla \zeta|^{2}+\zeta\left|\zeta_{\tau}\right|\right] w d x d \tau .
\end{aligned}
$$

In the similar way to (2.5) and (2.6), we have

$$
\begin{gathered}
\iint_{Q\left(\rho^{\prime}\right)}|c|^{2} u^{2} u_{l}^{r} \zeta^{2} w d x d \tau \leq 4 c_{1}^{2}\left(\sigma_{1} c_{3}\right)^{\frac{1}{\kappa^{\prime}}} \iint_{Q\left(\rho^{\prime}\right)}\left|\nabla\left(u u_{l}^{\frac{r}{2}} \zeta\right)\right|^{2} w d x d \tau \\
\leq 12 c_{1}^{2}\left(\sigma_{1} c_{3}\right)^{\frac{1}{\kappa^{\prime}}} \iint_{Q\left(\rho^{\prime}\right)}\left[\left(u_{l}^{r}|\nabla u|^{2}\right.\right. \\
\left.\left.+\frac{r^{2}}{4} u_{l}^{r}\left|\nabla u_{l}\right|^{2}\right) \zeta^{2}+u^{2} u_{l}^{r}|\nabla \zeta|^{2}\right] w d x d \tau
\end{gathered}
$$

In the similar way to $(2.8)$, for any $\nu>0$, we have

$$
\begin{gathered}
\iint_{Q\left(\rho^{\prime}\right)}\left[|d(x, \tau)|^{2}+|V(x, \tau)|\right] u^{2} u_{l}^{r} \zeta^{2} w d x d \tau \\
\leq \nu C_{4} \iint_{Q\left(\rho^{\prime}\right)}\left|\nabla\left(u u_{l}^{\frac{r}{2}} \zeta\right)\right|^{2} w d x d \tau \\
+C_{3} \iint_{Q\left(\rho^{\prime}\right)} u^{2} u_{l}^{r} \zeta^{2} w d x d \tau
\end{gathered}
$$


By (2.13)-(2.15), if $\sigma_{1}$ is sufficiently small such that

$$
\begin{aligned}
& 12 C_{1}\left(1+r^{-1}\right) c_{1}^{2}\left(\sigma_{1} c_{3}\right)^{\frac{1}{\kappa^{\prime}}}<\frac{1}{2} \\
& 3 C_{1}\left(1+r^{-1}\right) c_{1}^{2}\left(\sigma_{1} c_{3}\right)^{\frac{1}{\kappa^{\prime}}} r^{2}<\frac{1}{2},
\end{aligned}
$$

then we take a sufficiently small $\nu>0$, and have

$$
\begin{aligned}
& \sup _{-\left(\rho^{\prime}\right)^{2}<\tau<\left(\rho^{\prime}\right)^{2}} \int_{B\left(0,2 \rho^{\prime}\right)} u_{l}^{r+2} \zeta^{2} w d x \\
& +\iint_{Q\left(\rho^{\prime}\right)}\left[u_{l}^{r}|\nabla u|^{2}+r u_{l}^{r}\left|\nabla u_{l}\right|^{2}\right] \zeta^{2} w d x d \tau \\
& \leq 2 C_{5}^{\prime} \iint_{Q\left(\rho^{\prime}\right)} u^{2} u_{l}^{r}\left[\zeta^{2}+|\nabla \zeta|^{2}+\zeta\left|\zeta_{\tau}\right|\right] w d x d \tau
\end{aligned}
$$

for some constant $C_{5}^{\prime}>0$. Letting $l \rightarrow \infty$, if $u \in L_{\text {loc }}^{r+2}(Q, w d x d \tau)$, we have

$$
\begin{gathered}
\sup _{-\left(\rho^{\prime}\right)^{2}<\tau<\left(\rho^{\prime}\right)^{2}} \int_{B\left(0,2 \rho^{\prime}\right)} u^{r+2} \zeta^{2} w d x+\iint_{Q\left(\rho^{\prime}\right)}\left|\nabla\left[u^{\frac{r+2}{2}} \zeta\right]\right|^{2} w d x d \tau \\
\leq \frac{8 C_{5}^{\prime}}{\left(\rho^{\prime}-\rho\right)^{2}} \iint_{Q\left(\rho^{\prime}\right)} u^{r+2} w d x d \tau
\end{gathered}
$$

Therefore, by Lemma 2.2, if $u \in L_{\mathrm{loc}}^{r+2}(Q, w d x d \tau)$, then $u \in L_{\operatorname{loc}}^{(r+2) \bar{\kappa}}(Q$, $w d x d \tau)$.

By the definition of the solution of (1.1) and Lemma 2.2, $u \in L_{\text {loc }}^{2 \bar{\kappa}}(Q$, $w d x d \tau)$. Then, if $\sigma_{1}$ is a sufficiently small constant so that the equalities (2.16) hold with $r=2(\bar{\kappa}-1)>0$, we have $u \in L_{\text {loc }}^{\bar{\kappa}^{2}}(Q, w d x d \tau)$. Repeating this argument, if $\sigma_{1}$ is a sufficiently small constant, we have $u \in L_{\text {loc }}^{p}(Q, w d x d \tau)$, where $p$ is a constant given in Lemma 2.3. By Lemma 2.3 , we have $u \in L_{\text {loc }}^{\infty}(Q)$, and the proof of Lemma 2.4 is complete.

Lemma 2.5. Assume the same conditions as those of Proposition 2.1. Let $u$ be a nonnegative subsolution of (1.1) in $Q$. Then for any $p^{\prime} \in\left(0,1+\frac{\kappa^{\prime}}{\epsilon}\right]$, there exist positive constants $C$ and $\mu_{1}$ such that

$$
\|u\|_{L^{\infty}(Q(\rho))} \leq\left(C \frac{\left(\rho^{\prime}-\rho\right)^{-\frac{2}{\mu_{1}}}}{(w \otimes 1)\left(Q\left(\rho^{\prime}\right)\right)} \iint_{Q\left(\rho^{\prime}\right)} u^{p} w d x d \tau\right)^{\frac{1}{p}}
$$

for all $p^{\prime} \leq p \leq 2$ and $\frac{1}{2} \leq \rho<\rho^{\prime} \leq 1$. 
Proof of Lemma 2.5. For $\eta \in(0,1]$, we set $\rho_{n}=\rho+\eta 2^{-n}\left(\rho^{\prime}-\rho\right)$. We denote by $\zeta_{n}$ a nonnegative piecewise smooth function in $Q\left(\rho_{n}\right)=$ $B\left(0,2 \rho_{n}\right) \times\left(-\rho_{n}^{2}, \rho_{n}^{2}\right)$ such that $\zeta_{n}=1$ on $Q\left(\rho_{n+1}\right), \operatorname{supp} \zeta_{n} \subset Q\left(\rho_{n}\right)$, and

$$
\left|\nabla \zeta_{n}\right|^{2}+\left|\left(\zeta_{n}\right)_{\tau}\right| \leq 2^{2(n+1)} / \eta^{2}\left(\rho^{\prime}-\rho\right)^{2} .
$$

We multiply the equation (1.1) by $u_{k}^{r} \zeta_{n}^{2}, r=\frac{\kappa^{\prime}}{\epsilon}$. In the same way as in Lemma 2.3 , if $\sigma_{1}$ is sufficiently small, then we have

$$
\begin{aligned}
& \|u\|_{L^{\infty}\left(Q\left(\rho_{\infty}\right)\right)} \leq \\
& \quad\left(C_{8}^{-1} K^{\frac{1}{\mu_{1}}} \frac{1}{w\left(B\left(0,2 \rho_{0}\right)\right)} \iint_{Q\left(\rho_{0}\right)} u^{r+1} w d x d \tau\right)^{\frac{\mu_{1}}{(r+1)\left(1+\mu_{1}\right)}}
\end{aligned}
$$

where

$$
K=\left(\frac{1}{w\left(B\left(0,2 \rho_{0}\right)\right)} \iint_{Q\left(\rho_{0}\right)} u^{q(r+1)} w d x d \tau\right)^{\frac{1}{q}} \eta^{-2}\left(\rho^{\prime}-\rho\right)^{-2} .
$$

By Lemma 2.4, $u \in L_{\text {loc }}^{\infty}(Q)$. By (2.3) and (2.17), there exists a constant $C_{9}>0$ such that

$$
\begin{aligned}
& \quad\|u\|_{L^{\infty}\left(Q\left(\rho_{\infty}\right)\right)} \\
& \leq C_{9}\|u\|_{L^{\infty}\left(Q\left(\rho_{0}\right)\right)}^{\frac{1}{1+\mu_{1}}}\left(\frac{\left(\eta\left(\rho^{\prime}-\rho\right)\right)^{-\frac{2}{\mu_{1}}}}{(w \otimes 1)\left(Q\left(\rho^{\prime}\right)\right)} \iint_{Q\left(\rho^{\prime}\right)} u^{r+1} w d x d \tau\right)^{\frac{\mu_{1}}{(r+1)\left(1+\mu_{1}\right)}} \\
& \leq C_{9}\|u\|_{L^{\infty}\left(Q\left(\rho_{0}\right)\right)}^{\frac{(r+1-p) \mu_{1}+r+1}{(r+1)\left(1+\mu_{1}\right)}}\left(\frac{\left(\eta\left(\rho^{\prime}-\rho\right)\right)^{-\frac{2}{\mu_{1}}}}{(w \otimes 1)\left(Q\left(\rho^{\prime}\right)\right)} \iint_{Q\left(\rho^{\prime}\right)} u^{p} w d x d \tau\right)^{\frac{\mu_{1}}{(r+1)\left(1+\mu_{1}\right)}}
\end{aligned}
$$

for any $p \in\left(0,1+\frac{\kappa^{\prime}}{\epsilon}\right)$.

Next we use the method of iteration with respect to $\eta$. Put $\eta_{s}=1-$ $\sum_{i=1}^{s} 2^{-i+1}, s=1,2, \ldots$ Set $Q_{s}=Q\left(\rho^{\prime}-\eta_{s}\left(\rho^{\prime}-\rho\right)\right)$ and $X_{s}=\|u\|_{L^{\infty}\left(Q_{s}\right)}$. Applying (2.21) to the pair of $Q_{s} \subset Q_{s+1}$, we obtain

$$
X_{s} \leq C_{9}\left\{2^{\frac{2 s}{\mu_{1}}} X_{s+1}^{a-p} \frac{\left(\rho^{\prime}-\rho\right)^{-\frac{2}{\mu_{1}}}}{(w \otimes 1)\left(Q\left(\rho^{\prime}\right)\right)} \iint_{Q\left(\rho^{\prime}\right)} u^{p} w d x d \tau\right\}^{\frac{1}{a}}, \quad s=1,2 \ldots
$$

where $a=\left(\frac{\kappa^{\prime}}{\epsilon}+1\right) \frac{1+\mu_{1}}{\mu_{1}}$. By the Young inequality, for any $\nu>0$, there exists a constant $C_{10}>0$ such that $X_{s} \leq \nu X_{s+1}+C_{10} 2^{\frac{2 s}{\mu_{1} p}}\left(\frac{\left(\rho^{\prime}-\rho\right)^{-\frac{2}{\mu_{1}}}}{(w \otimes 1)\left(Q\left(\rho^{\prime}\right)\right)} \iint_{Q\left(\rho^{\prime}\right)} u^{p} w d x d \tau\right)^{\frac{1}{p}}, \quad s=1,2 \ldots$ 
Iteration of these inequalities yields

$$
X_{1} \leq \nu^{s} X_{s+1}+C_{10} \sum_{i=1}^{s} \nu^{i} 2^{\frac{2 i}{\mu_{1} p}}\left(\frac{\left(\rho^{\prime}-\rho\right)^{-\frac{2}{\mu_{1}}}}{(w \otimes 1)\left(Q\left(\rho^{\prime}\right)\right)} \iint_{Q\left(\rho^{\prime}\right)} u^{p} w d x d \tau\right)^{\frac{1}{p}}
$$

Choosing $\nu=2^{\frac{2}{\mu_{1} p}-1}$ and taking the limit $s \rightarrow \infty$, we obtain the inequality

$$
\|u\|_{L^{\infty}(Q(\rho))} \leq C_{11}\left(\frac{\left(\rho^{\prime}-\rho\right)^{-\frac{2}{\mu_{1}}}}{(w \otimes 1)\left(Q\left(\rho^{\prime}\right)\right)} \iint_{Q\left(\rho^{\prime}\right)} u^{p} w d x d \tau\right)^{\frac{1}{p}}
$$

where $C_{11}$ is a constant depending on $p$. So the proof of Lemma 2.5 is complete.

LEMma 2.6. Assume the same conditions as those of Proposition 2.1. Let $u$ be a nonnegative supersolution in $Q$. Then then there exist positive constants $C$ and $\mu_{2}$ such that for any $p \in\left(0, \frac{1}{\bar{\kappa}}\right)$, there exists a positive constant $p^{\prime} \in\left(\frac{1}{\bar{\kappa}}, 1\right)$ such that

$$
\begin{aligned}
\left(\frac{1}{(w \otimes 1)(Q(\rho))} \iint_{Q(\rho)} u^{p^{\prime}} w d x d \tau\right)^{\frac{1}{p^{\prime}}} \leq \\
\left(\frac{C\left(\rho^{\prime}-\rho\right)^{-2 \mu_{2}}}{(w \otimes 1)\left(Q\left(\rho^{\prime}\right)\right)} \iint_{Q\left(\rho^{\prime}\right)} u^{p} w d x d \tau\right)^{\frac{1}{p}}
\end{aligned}
$$

for all $\frac{1}{2} \leq \rho<\rho^{\prime} \leq 1$

Proof of Lemma 2.6. Let $v(x, t)=u(x,-t),(x, t) \in Q$. In the same way as in Lemma 2.4, we have

$$
\begin{aligned}
& \frac{1}{r+1} \sup _{-\left(\rho^{\prime}\right)^{2}<\tau<\left(\rho^{\prime}\right)^{2}} \int_{B\left(0,2 \rho^{\prime}\right)} v^{r+1} \zeta^{2} w d x \\
& +\frac{|r|}{(r+1)^{2}} \iint_{Q\left(\rho^{\prime}\right)}\left|\nabla\left[v^{\frac{r+1}{2}} \zeta\right]\right|^{2} w d x d \tau \\
& \leq C_{1}\left(1+|r|^{-1}\right) \iint_{Q\left(\rho^{\prime}\right)}\left[\left(|c(x, \tau)|^{2}+|d(x, \tau)|^{2}\right) v^{r+1} \zeta^{2}+|V| v^{r+1} \zeta^{2}\right] w d x d \tau \\
& \quad+C_{1}\left(1+|r|^{-1}+(1+r)^{-1}\right) \iint_{Q\left(\rho^{\prime}\right)} v^{r+1}\left[|\nabla \zeta|^{2}+\zeta\left|\zeta_{\tau}\right|\right] w d x d \tau
\end{aligned}
$$


for $r \in(-1,0)$. Let $-1<r<\frac{1}{\bar{\kappa}}-1$. In the same way as $(2.9)$, if $\sigma$ is sufficiently small, then there exists a constant $C_{12}>0$ such that

$$
\begin{aligned}
\sup _{-\left(\rho^{\prime}\right)^{2}<\tau<\left(\rho^{\prime}\right)^{2}} \int_{B\left(0,2 \rho^{\prime}\right)} & v^{r+1} \zeta^{2} w d x+\iint_{Q\left(\rho^{\prime}\right)}\left|\nabla\left[v^{\frac{r+1}{2}} \zeta\right]\right|^{2} w d x d \tau \\
& \leq C_{12} \iint_{Q\left(\rho^{\prime}\right)} v^{r+1}\left[1+|\nabla \zeta|^{2}+\zeta\left|\zeta_{\tau}\right|\right] w d x d \tau .
\end{aligned}
$$

Here the constant $C_{12}$ is independent of $r$. We remark that, by $-1<r<$ $\frac{1}{\bar{\kappa}}-1$, there exists a constant $\sigma_{0}$ independent of $r$ such that if $\sigma \leq \sigma_{0}$, then the inequality (2.23) holds. By Lemma 2.2 and (2.23), we have

$$
\begin{aligned}
& \left(\frac{1}{(w \otimes 1)(Q(\rho))} \iint_{Q(\rho)} v^{\bar{\kappa}(r+1)} w d x d \tau\right)^{\frac{1}{\bar{\kappa}(r+1)}} \\
& \leq\left(C_{13} \frac{\left(\rho^{\prime}-\rho\right)^{-2}}{(w \otimes 1)\left(Q\left(\rho^{\prime}\right)\right)} \iint_{Q\left(\rho^{\prime}\right)} v^{r+1} w d x d \tau\right)^{\frac{1}{r+1}}
\end{aligned}
$$

for some constant $C_{13}>0$.

Let $p$ be a positive constant such that $p \in\left(0, \frac{1}{\bar{\kappa}}\right)$. Let $n \in \mathbb{N} \cup\{0\}$ such that $\bar{\kappa}^{n} p<\frac{1}{\bar{\kappa}}$ and $\bar{\kappa}^{n+1} p \geq \frac{1}{\bar{\kappa}}$. Set $\rho_{j}=\rho+2^{-j}\left(\rho^{\prime}-\rho\right)$ and

$$
z_{j}=\left(\frac{1}{(w \otimes 1)\left(Q\left(\rho_{j}\right)\right)} \iint_{Q\left(\rho_{j}\right)} v^{\bar{\kappa}^{j} p} w d x d \tau\right)^{\frac{1}{\bar{\kappa}^{j} p}}, \quad j=0, \ldots, n .
$$

Applying (2.24) to the pair of $Q\left(\rho_{j+1}\right) \subset Q\left(\rho_{j}\right)$, we have

$$
z_{j+1} \leq\left(C_{13} 2^{3(j+1)}\left(\rho^{\prime}-\rho\right)^{-2}\right)^{\frac{1}{\bar{\kappa}^{j} p}} z_{j}, \quad j=0, \ldots, n .
$$

By (2.25),

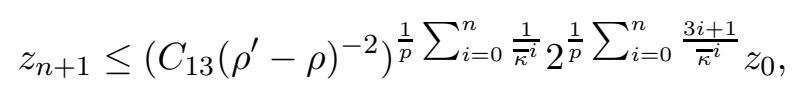

and so there exist constants $\mu_{2}$ and $C_{14}$ independent of $p$ such that

$$
z_{n+1} \leq\left(C_{14}\left(\rho^{\prime}-\rho\right)^{-2 \mu_{2}}\right)^{\frac{1}{p}} z_{0} .
$$

Furthermore, by (2.5),

$$
\left(\frac{1}{(w \otimes 1)(Q(\rho))} \iint_{Q(\rho)} v^{\bar{\kappa}^{n+1} p} w d x d \tau\right)^{\frac{1}{\bar{\kappa}^{n+1} p}} \leq c_{3}^{\frac{1}{\bar{\kappa}^{n+1} p}} z_{n+1} .
$$

Since $\bar{\kappa}^{n+1} p \geq \frac{1}{\bar{\kappa}}$, by $(2.26)$ and $(2.27)$, we obtain the inequality $(2.22)$, and so the proof of Lemma 2.6 is complete.

By Lemmas 2.5 and 2.6, we have Proposition 2.1. 


\section{$\S 3$. Interior Harnack inequality}

In this section we give the interior Harnack inequality for the degenerate parabolic equation (1.1) by using Proposition 2.1. Furthermore, we obtain some inequalities and prove the continuity of solutions of (1.1).

Theorem 3.1. Let $Q \subset D$. Assume (A1)-(A3) and (A4.a). Let $u$ be a nonnegative solution of (1.1) in $Q$. If $\sigma \leq \sigma_{1}$, then there exists a constant C such that

$$
\sup _{Q^{-}} u \leq C \inf _{Q^{+}} u .
$$

Here $\sigma_{1}$ is the constant given in Proposition 2.1 and $C$ depends only on $N$, $\lambda, c_{0}, \epsilon$, and $\mathcal{D}$.

In order to prove Theorem 3.1, by Proposition 2.1 and the arguments of [CS1,2,3], we have only to prove the following lemmas.

Lemma 3.2. Assume the same conditions as those of Theorem 3.1. Let $u$ be a nonnegative solution of (1.1) in $Q$. Then there exist positive constants $C_{1}$ and $a$ such that

$$
\begin{aligned}
& (w \otimes 1)\left(\left\{(x, t) \in Q^{+} \mid \log u(x, t)<-s+a\right\}\right) \\
& \quad+(w \otimes 1)\left(\left\{(x, t) \in Q^{-} \mid \log u(x, t)>s+a\right\}\right) \leq \frac{C_{1}}{s} w(B(0,1)) .
\end{aligned}
$$

Here the constant a depends on $u$.

Lemma 3.3. Let $\mu, C_{2}$, and $\theta \in\left[\frac{1}{2}, 1\right)$ be some positive constants. Let $v$ be a positive function defined in a neighborhood of $Q$ such that

$$
\sup _{Q(\rho)} v \leq\left[\frac{C_{2}}{\left(\rho^{\prime}-\rho\right)^{\mu}(w \otimes 1)\left(Q\left(\rho^{\prime}\right)\right)} \int_{Q\left(\rho^{\prime}\right)} v^{p} w d x d \tau\right]^{\frac{1}{p}}
$$

for all $\rho, \rho^{\prime}$, and $p$ such that $\frac{1}{2} \leq \theta \leq \rho<\rho^{\prime} \leq 1,0<p<2$.

Moreover assume that

$$
(w \otimes 1)(\{(x, t) \in Q \mid \log v>s\}) \leq \frac{C_{2}}{s}(w \otimes 1)(Q), \quad s>0 .
$$

Then there exists a constant $\gamma$ such that

$$
\sup _{Q(\theta)} v<\gamma
$$


In the same way as in $[\mathrm{CS} 1,2,3]$, we can prove Lemmas 3.2 and 3.3. Therefore, we obtain Theorem 3.1 .

Proof of Theorem 3.1. By Proposition 2.1, Lemma 3.2, and Lemma 3.3, there exists a positive constant $\gamma_{1}$ such that

$$
\sup _{Q^{-}} e^{-a} u<\gamma_{1} .
$$

On the other hand, applying Lemma 2.5 to the function $v(x, t)=u^{-p}(x, t)$, $p>0$, we see that the inequality $(2.1)$ holds with $u$ replaced by $u^{-1}$. By Lemmas 3.2 and 3.3, there exists a positive constant $\gamma_{2}$ such that

$$
\sup _{Q^{+}} e^{a} u^{-1}<\gamma_{2}
$$

Therefore, we have

$$
\sup _{Q^{-}} u(x, t) \leq \gamma_{1} \gamma_{2} \inf _{Q^{+}} u(x, t)
$$

and the proof of Theorem 3.1 is complete.

Proof of Theorem A. By Theorem 3.1, it suffices to prove that (A4.a) holds. By (A4), for any $\alpha>0$, there exist an integer $n$ and a sequence $\left\{t_{j}\right\}_{j=0}^{n}$ with $-1 \leq t_{1}<t_{2}<\ldots<t_{n} \leq 1$ such that

$$
\left\|c(\cdot, t)-c\left(\cdot, t_{j}\right)\right\|_{L^{2 \kappa^{\prime}}(\Omega, w d x)} \leq \alpha, \quad t_{j} \leq t \leq t_{j+1}, \quad j=0,1, \ldots, n-1 .
$$

Furthermore, for any $j$, there exists an $L^{2\left(\kappa^{\prime}+\epsilon\right)}(\Omega, w d x)$-function $\tilde{c}_{j}$ such that

$$
\left\|c\left(\cdot, t_{j}\right)-\tilde{c}_{j}(\cdot)\right\|_{L^{2 \kappa^{\prime}}(\Omega, w d x)} \leq \alpha
$$

Put

$$
\tilde{c}(x, t)=\tilde{c}_{j}(x, t), \quad x \in \Omega, \quad t_{j} \leq t \leq t_{j+1}, \quad j=0,1, \ldots, n-1 .
$$

Then we have

$$
\sup _{-1<t<1}\|c(\cdot, t)-\tilde{c}(\cdot, t)\|_{L^{2 \kappa^{\prime}}(\Omega, w d x)} \leq 2 \alpha
$$

This together with the arbitrariness of $\alpha$ implies the condition (A4.a). So the proof of Theorem A is complete. 
Next we consider the inhomogeneous degenerate parabolic equation

$$
\begin{aligned}
\frac{\partial}{\partial t} u=\frac{1}{\omega(x)} \sum_{i, j=1}^{N} \frac{\partial}{\partial x_{j}}( & \left.a_{i j}(x, t) \omega(x) \frac{\partial}{\partial x_{i}} u\right) \\
& +\sum_{i=1}^{N} b_{i}(x, t) \frac{\partial}{\partial x_{i}} u-V(x, t) u+f(x, t)
\end{aligned}
$$

in $D$, where $f \in L^{\infty}\left((-1,1): L^{\kappa^{\prime}+\epsilon}(\Omega, w d x)\right)$. Let $Q \subset D$ and set

$$
v=u+F, \quad F=\sup _{-1<t<1}\left(f_{B(0,2)}|f(x, t)|^{\kappa^{\prime}+\epsilon} w(x) d x\right)^{\frac{1}{\kappa^{\prime}+\epsilon}}
$$

Then we have

$$
|-V u+f| \leq\left(|V|+F^{-1}|f|\right)|v|
$$

and

$$
\begin{array}{r}
\text { (3.8) } \sup _{-1<t<1}\left(f_{B(0,2)}\left[|V(x, t)|+F^{-1}|f(x, t)|\right]^{\kappa^{\prime}+\epsilon} w(x) d x\right)^{\frac{1}{\kappa^{\prime}+\epsilon}} \\
\leq \sup _{-1<t<1}\left(f_{B(0,2)}|V(x, t)|^{\kappa^{\prime}+\epsilon} w(x) d x\right)^{\frac{1}{\kappa^{\prime}+\epsilon}}+1 .
\end{array}
$$

By (3.7) and (3.8), we apply the same argument as in the proof of Theorem 3.1 to the function $v$, and obtain the following theorem (see also [Se]).

Theorem 3.4. Let $Q \subset D$. Assume (A1)-(A3) and (A4.a). Let $f \in$ $L^{\infty}\left((-1,1): L^{\kappa^{\prime}+\epsilon}(\Omega, w d x)\right)$. Let $u$ be a nonnegative solution of $(3.5)$ in $Q$. If $\sigma \leq \sigma_{1}$, then there exists a constant $C$ such that

$$
\sup _{Q^{-}}[u+F] \leq C \inf _{Q^{+}}[u+F]
$$

where $F$ is a constant given in (3.6). Here $C$ depends only on $N, \lambda, c_{0}, \epsilon$, and $\mathcal{D}$.

Next, we give more general result than that of Theorem 3.1.

TheOrem 3.5. Assume (A1)-(A3), (A5), and the following condition: (A4.b) There exist a constant $\sigma$ and measurable functions $c$ and d defined on $D$ and such that 
(i) $b(x, t)=c(x, t)+d(x, t) \quad$ for almost all $(x, t) \in D$,

(ii) $\sup _{-1<t<1} \int_{\Omega}|c(x, t)|^{2 \kappa^{\prime}} w(x) d x \leq \sigma c_{2}, \quad x \in \Omega$,

(iii) $d \in L^{\infty}\left((-1,1): L^{2\left(\kappa^{\prime}+\epsilon\right)}(\Omega, w d x)\right)$.

Let $u$ be a nonnegative solution of (1.1) in D. Let $\Omega^{\prime}$ be a convex subdomain of $\Omega$ and set $d=\operatorname{dist}\left(\Omega^{\prime}, \partial \Omega\right)$. Then there exists a constant $\sigma_{2}=\sigma_{2}\left(N, c_{0}, \epsilon\right)$ such that, if $\sigma \leq \sigma_{2}$, there exists a constant $C>0$ such that

$$
\begin{aligned}
u(y, s) \leq u(x, t) \exp \left[C\left(\frac{|x-y|^{2}}{t-s}+\frac{t-s}{k}+1\right)\right], & \\
& k=\min \left\{1, s+1, d^{2}\right\},
\end{aligned}
$$

for all $x, y \in \Omega^{\prime}$ and all $s, t$ with $-1<s<t<1$. Here $C$ depends only on $N, \lambda, c_{0}, \epsilon$, and $c_{2} \mathcal{E}(\Omega)$, where

$$
\mathcal{E}(\Omega)=\sup _{-1<t<1} \int_{\Omega}\left[|d(x, t)|^{2}+|V(x, t)|\right]^{\kappa^{\prime}+\epsilon} w(x) d x .
$$

Proof of Theorem 3.5. Let $\left(x_{0}, t_{0}\right) \in D$ and $\rho \in\left(0, \frac{1}{2}\right)$ with $Q_{x_{0}, t_{0}}(\rho) \subset$ $D$. For any measurable function $g$ defined on $Q_{x_{0}, t_{0}}(\rho)$, we set

$$
\tilde{g}(y, s)=g\left(x_{0}+\rho y, t_{0}+\rho^{2} s\right), \quad(y, s) \in Q .
$$

Then $\tilde{u}$ satisfies the degenerate parabolic equation

$$
\begin{aligned}
\frac{\partial}{\partial s} \tilde{u}=\frac{1}{\tilde{w}(y)} \sum_{i, j=1}^{N} \frac{\partial}{\partial y_{j}}( & \left.\tilde{a}_{i j}(y, s) \tilde{w}(y) \frac{\partial}{\partial y_{i}} \tilde{u}\right) \\
& +\sum_{i=1}^{N} \rho \tilde{b}_{i}(y, s) \frac{\partial}{\partial y_{i}} \tilde{u}-\rho^{2} \tilde{V}(y, s) \tilde{u}
\end{aligned}
$$

in $Q$. Then by (A4.b) and (A5),

$$
\sup _{-1<s<1} f_{B(0,2)}|\rho \tilde{c}(y, s)|^{2 \kappa^{\prime}} \tilde{w}(y) d y \leq \frac{\sigma_{2}}{2^{\kappa^{\prime}}} .
$$

Furthermore, by (A5), there exists a constant $C$ independent of $x_{0}$ and $\rho$ such that

$$
\begin{aligned}
(3.12) & \sup _{-1<s<1} f_{B(0,2)}\left[|\rho \tilde{d}(x, s)|^{2}+\left|\rho^{2} \tilde{V}(x, s)\right|\right]^{\kappa^{\prime}+\epsilon} \tilde{w}(x) d x \\
& \leq \frac{\rho^{2 \epsilon}}{2^{\kappa^{\prime}+\epsilon} c_{2}} \sup _{-1<t<1} \int_{\Omega}\left[|d(x, t)|^{2}+|V(x, t)|\right]^{\kappa^{\prime}+\epsilon} w(x) d x \leq C \rho^{2 \epsilon}
\end{aligned}
$$


If necessary, we take a sufficiently small $\sigma_{2}$ so that $\sigma_{2} \leq 2^{\kappa^{\prime}} \sigma_{1}$. By (3.11) and (3.12), we apply Theorem 3.1 to the function $v$, and obtain

$$
\sup _{Q_{x_{0}, t_{0}}^{-}(\rho)} u \leq C \inf _{Q_{x_{0}, t_{0}}^{+}(\rho)} u
$$

where $C$ is a constant independent of $x_{0}$ and $\rho$. By (3.13), we apply the same argument as in $[\mathrm{Ms}]$ to the function $u$, and obtain the inequality (3.9). So the proof of Theorem 3.5 is complete.

By Theorem 3.4, we obtain the continuity of solutions of (1.1).

TheOrem 3.6. Assume (A1)-(A3), (A4.b), and (A5). Let u be a solution of (1.1) in D. If $\sigma \leq \sigma_{2}$, then there exist positive constants $C$ and $\delta$ such that

$$
\underset{Q_{x, t}(\rho)}{\operatorname{Osc}} u \leq C\left(\frac{\rho}{\rho_{1}}\right)^{\delta}\left(\underset{Q_{x, t}\left(\rho_{1}\right)}{\operatorname{osc}} u+\rho_{1}^{\frac{2 \epsilon}{\kappa^{\prime}+\epsilon}}\|u\|_{L^{\infty}\left(Q_{x, t}\left(\rho_{1}\right)\right)}\right)
$$

for all $0<\rho<\rho_{1} \leq \frac{1}{2}$ with $Q_{x, t}\left(\rho_{1}\right) \subset D$. Here $C$ and $\delta$ depend only on $N$, $\lambda, c_{0}, \epsilon$, and $c_{2} \mathcal{E}(\Omega)$.

Proof of Theorem 3.6. Let $\left(x_{0}, t_{0}\right) \in D$ and $\rho \in\left(0, \frac{1}{2}\right)$ with $Q_{x_{0}, t_{0}}(\rho) \subset$ $D$. In the same way as in the proof of Theorem 3.5, $\tilde{u}$ satisfies the parabolic equation (3.10) in $Q$. Set

$$
\tilde{u}^{+}(x, t)=\sup _{Q} \tilde{u}-\tilde{u}(x, t), \quad \tilde{u}^{-}(x, t)=\tilde{u}(x, t)-\inf _{Q} \tilde{u} .
$$

Since $\tilde{u}^{+}$and $\tilde{u}^{-}$are nonnegative functions, by (3.6), we apply Theorem 3.4 to the functions $\tilde{u}^{+}$and $\tilde{u}^{-}$. Then there exists a constant $C_{3}$ such that

$$
\begin{aligned}
& \sup _{Q^{-}}\left(\tilde{u}^{+}+F_{1}\right) \leq C_{3} \inf _{Q^{+}}\left(\tilde{u}^{+}+F_{1}\right), \\
& \sup _{Q^{-}}\left(\tilde{u}^{-}+F_{2}\right) \leq C_{3} \inf _{Q^{+}}\left(\tilde{u}^{-}+F_{2}\right),
\end{aligned}
$$

where

$$
\begin{aligned}
& F_{1}=\left|\sup _{Q} \tilde{u}\right| \sup _{-1<s<1}\left(f_{B(0,2)}\left|\rho^{2} \tilde{V}(y, s)\right|^{\kappa^{\prime}+\epsilon} \tilde{w}(y) d y\right)^{\frac{1}{\kappa^{\prime}+\epsilon}} \\
& F_{2}=\left|\inf _{Q} \tilde{u}\right| \sup _{-1<s<1}\left(f_{B(0,2)}\left|\rho^{2} \tilde{V}(y, s)\right|^{\kappa^{\prime}+\epsilon} \tilde{w}(y) d y\right)^{\frac{1}{\kappa^{\prime}+\epsilon}} .
\end{aligned}
$$


By (3.12), (3.17), and (3.18), there exists a constant $C_{4}$ such that

$$
F_{1} \leq C_{4}\left|\sup _{Q} \tilde{u}\right| \rho^{\frac{2 \epsilon}{\kappa^{\prime}+\epsilon}}, \quad F_{2} \leq C_{4}\left|\inf _{Q} \tilde{u}\right| \rho^{\frac{2 \epsilon}{\kappa^{\prime}+\epsilon}}
$$

On the other hand,

$$
\frac{1}{(\tilde{w} \otimes 1)\left(Q^{-}\right)} \iint_{Q^{-}}\left(\tilde{u}^{+}+F_{1}\right) \tilde{w}(x) d x \leq \sup _{Q^{-}}\left(\tilde{u}^{+}+F_{1}\right)
$$

$$
\frac{1}{(\tilde{w} \otimes 1)\left(Q^{-}\right)} \iint_{Q^{-}}\left(\tilde{u}^{-}+F_{2}\right) \tilde{w}(x) d x \leq \sup _{Q^{-}}\left(\tilde{u}^{-}+F_{2}\right) .
$$

By (3.16), (3.20) and (3.21),

$$
\underset{Q}{\operatorname{Osc}} \tilde{u}+F_{1}+F_{2} \leq C_{3}\left(\underset{Q}{\operatorname{Osc}} \tilde{u}-\underset{Q^{+}}{\operatorname{osc}} \tilde{u}+F_{1}+F_{2}\right)
$$

Set $\theta=\frac{C_{3}-1}{C_{3}} \in(0,1)$. By $(3.22)$, there exists a constant $C_{4}$ such that

$$
\begin{aligned}
& \underset{Q_{x_{0}, t_{0}}^{+}(\rho)}{\operatorname{Osc}} u \leq \theta\left(\underset{Q_{x_{0}, t_{0}}(\rho)}{\operatorname{Osc}} u+F_{1}+F_{2}\right) \\
& \leq \theta\left\{\operatorname{osc}_{Q_{x_{0}, t_{0}}(\rho)}^{\operatorname{osc}} u+C_{4} \rho^{\frac{2 \epsilon}{\kappa^{\prime}+\epsilon}}\left(\left|\sup _{Q_{x_{0}, t_{0}}(\rho)} u\right|+\left|\inf _{Q_{x_{0}, t_{0}}(\rho)} u\right|\right)\right\} .
\end{aligned}
$$

By (3.23) and the arbitrariness of $t_{0}$, we have

$$
\begin{aligned}
\underset{Q_{x_{0}, t_{0}}\left(\frac{\rho}{3}\right)}{\operatorname{Osc}} u & \leq \theta\left\{\operatorname{Osc}_{Q_{x_{0}, t_{0}}(\rho)}^{\operatorname{Osc}} u+C_{4} \rho^{\frac{2 \epsilon}{\kappa^{\prime}+\epsilon}}\left(\left|\sup _{Q_{x_{0}, t_{0}}(\rho)} u\right|+\left|\inf _{Q_{x_{0}, t_{0}}(\rho)} u\right|\right)\right\} \\
& \leq \theta\left\{\operatorname{OSc}_{Q_{x_{0}, t_{0}}(\rho)}^{\operatorname{Osc}} u+2 C_{4} \rho^{\frac{2 \epsilon}{\kappa^{\prime}+\epsilon}}\|u\|_{L^{\infty}\left(Q_{x_{0}, t_{0}}(\rho)\right)}\right\} .
\end{aligned}
$$

By (3.24) and the similar way to the argument of Theorem 2.2 in [T], we get (3.14), and so the proof of Theorem 3.6 is complete.

In the similar way as in the proof of Theorem A, we see that (A4) implies (A4.b). Therefore, by Theorem 3.6, we have Theorem B. 


\section{$\S 4$. Boundary Harnack Inequality}

In this section we modify the argument of [Sa], and obtain the boundary Harnack inequality of nonnegative solutions of (1.1).

THEOREM 4.1. Let $x_{0} \in \partial \Omega$. Assume that there exist a positive constant $r_{0}$ and an orthonormal system such that $\Omega \cap B\left(x_{0}, r_{0}\right)$ is described as (1.6) and (1.7). Assume (A1)-(A3), (A5), and the following condition:

(A4.c) There exist a constant $\sigma$ and measurable functions $c$ and $d$ defined on $D$ such that

(i) $b(x, t)=c(x, t)+d(x, t) \quad$ for almost all $(x, t) \in\left(\Omega \cap B\left(x_{0}, r_{0}\right)\right) \times$ $(-1,1)$,

(ii) $\sup _{-1<t<1} \int_{\Omega \cap B\left(x_{0}, r_{0}\right)}|c(x, t)|^{2 \kappa^{\prime}} w(x) d x \leq \sigma c_{2}, \quad x \in \Omega$,

(iii) $\left.d \in L^{\infty}\left((-1,1): L^{2\left(\kappa^{\prime}+\epsilon\right)}\left(\Omega \cap B\left(x_{0}, r_{0}\right), w d x\right)\right)\right)$.

Let $u$ be a nonnegative solution of (1.1) in $D$ vanishing continuously on $\left[\partial \Omega \cap B\left(x_{0}, r_{0}\right)\right] \times(-1,1)$. Then there exists a constant $\sigma_{3}=\sigma_{3}\left(N, c_{0}, \epsilon, m\right)$ such that, if $\sigma \leq \sigma_{3}$, there exists a positive constant $C$ such that

$$
\begin{aligned}
& u(x, t) \leq C u\left(\left(x_{0}^{\prime}, \varphi\left(x_{0}^{\prime}\right)+r\right), s+2 r^{2}\right), \\
& s \in(-1,1), r<\frac{1}{4} \min \left\{r_{0}, 2 \sqrt{1-|s|}\right\},
\end{aligned}
$$

for $(x, t) \in D \cap\left\{(x, t) \in \mathbf{R}^{N+1}|| x-x_{0}|<r| t-s \mid,<r^{2}\right\}$. Here $C$ depends only on $N, \lambda, c_{0}, \epsilon, m$, and $c_{2} \mathcal{E}\left(\Omega \cap B\left(x_{0}, r_{0}\right)\right)$.

Before starting the proof of Theorem 4.1, we introduce some notations. Let

$$
\begin{aligned}
\Phi & =\left\{\left(x^{\prime}, x_{N}\right) \in \mathbf{R}^{N}\left|0<x_{N}<8,\right| x_{i} \mid<4, i=1,2, \ldots N-1\right\}, \\
\Phi^{\prime} & =\left\{\left(x^{\prime}, x_{N}\right) \in \mathbf{R}^{N}\left|0<x_{N}<2,\right| x_{i} \mid<2, i=1,2, \ldots N-1\right\},
\end{aligned}
$$

$\Psi=\Phi \times(-2,2)$, and $\Psi^{\prime}=\Phi^{\prime} \times\left(-\frac{1}{8}, \frac{1}{8}\right)$. Furthermore, we set

$$
v(x, t)=u\left(\left(x_{0}^{\prime}, \varphi\left(x_{0}^{\prime}\right)\right)+\frac{r}{2} x, s+8 r^{2} t\right), \quad(x, t) \in \Psi .
$$

Then $v$ satisfies the following degenerate parabolic parabolic equation

$$
\begin{aligned}
\frac{\partial}{\partial t} v=\frac{1}{\tilde{w}(x)} \sum_{i, j=1}^{N} \frac{\partial}{\partial x_{j}}\left(\tilde{a}_{i j}(x, t) \tilde{w}(x) \frac{\partial}{\partial x_{i}} v\right) & \\
& +\sum_{i=1}^{N} \tilde{b}_{i}(x, t) \frac{\partial}{\partial x_{i}} v-\tilde{V}(x, t) v
\end{aligned}
$$


in $\Psi$. Here, by the assumptions of Theorem 4.1, the coefficients $\left\{\tilde{a}_{i j}(x, t)\right\}_{i, j=1}^{N}, \tilde{w}$ satisfy the conditions (A1)-(A3), and (A5), respectively. Furthermore, if necessary, we take a sufficiently small $\sigma_{3}$ so that the condition (A4.b) holds with $b$ replaced by $\tilde{b}$.

In the same way as in $[\mathrm{Sa}]$, we set

$$
\begin{aligned}
& Q_{k, h, j}= \\
& \left\{\left(x^{\prime}, x_{N}\right) \in \mathbf{R}^{N} \mid \frac{1}{2^{k}}<x_{n}<\frac{1}{2^{k-1}}, \frac{h}{2^{k-1}}<x_{i}<\frac{h+1}{2^{k-1}}, i=1, \ldots, N-1\right\} \\
& \times\left(-\frac{1}{8}+\frac{j}{4^{k+2}},-\frac{1}{8}+\frac{j+1}{4^{k+2}}\right],
\end{aligned}
$$

where $k=1,2, \cdots, h=-2^{k-1}, \ldots, 2^{k-1}-1$, and $j=0,1, \ldots, 2^{2 k+2}-1$. By Theorem 3.5, there exists a constant $H_{1}$ such that

$$
v(x, t) \leq H_{1} v\left(P_{k, h, j}\right), \quad(x, t) \in Q_{k, h, j},
$$

where $P_{k, h, j}$ is the point whose coordinates are

$$
x_{i}=\frac{2 h+1}{2^{k}}, i=1,2, \ldots, N-1, \quad x_{n}=\frac{1}{2^{k-1}}, \quad t=-\frac{1}{8}+\frac{j+1}{4^{k+2}}+\frac{1}{4^{k} \cdot 8} .
$$

Moreover, if we consider for each $k$ and each $h=-2^{k-1}, \ldots, 2^{k-1}-1$, the point $P_{k, h, j}$, with $j=2^{k+2}-1$, whose $t$-coordinate is $\frac{1}{8}+\frac{\nu}{4^{k}}$, we see, again by Theorem 3.5 , that

$$
v\left(P_{k, h, j}\right) \leq H_{2}^{k-1} v(\bar{P}), \quad \bar{P}=\left(X_{0}, T_{0}\right)=\left(0,2, \frac{1}{4}\right) .
$$

By (4.4) and (4.5), we set $H=\max \left\{H_{1}, H_{2}\right\}$, and obtain

$$
v(x, t) \leq H^{5 k-1} v(\bar{P})
$$

for all $(x, t) \in Q_{k, h, j}$ and all $Q_{k, h, j}$. Furthermore, if $(x, t) \in Q_{k, h, j}$, then $x_{N}<2^{2-k}$.

Proof of Theorem 4.1. Reflecting $v$ across $x_{N}=0$ as an odd function of $x_{N}$, we obtain a function $\tilde{v}$, which is a weak solution of (4.3) in

$$
\tilde{\Psi}=\left\{\left(x^{\prime}, x_{N}\right) \in \mathbf{R}^{N}|| x_{N}|<8,| x_{i} \mid<4, i=1, \ldots, N-1\right\} \times(-2,2) .
$$


Assume that $P_{0}=\left(x_{0}, t_{0}\right)=\left(x_{0}^{\prime}, x_{0 N}, t_{0}\right)$ is a point in $\Psi^{\prime}$ such that $v\left(P_{0}\right)>H^{5 h_{0}} v(\bar{P})$, where $h_{0}$ is a constant to be chosen later. Set

$$
E_{P_{0}}=Q_{P_{0}}\left(2^{2-h_{0}}\right), \quad E_{P_{0}}^{n}=Q_{P_{0}}\left(2^{2-h_{0}+2 n}\right),
$$

where $n$ is a positive integer to be chosen later. If necessary, we take a sufficiently large $h_{0}$ so that $E_{P_{0}}^{n} \subset \Psi$. By (4.6), for any $l \in\{0,1, \ldots, n\}$, $\sup _{E_{P_{0}}^{l}} v>0$ and $\inf _{E_{P_{0}}^{l}} v<0$. So we have

$$
\left|\sup _{E_{P_{0}}^{l}} v\right|+\left|\inf _{E_{P_{0}}^{l}} v\right| \leq 2 \underset{E_{P_{0}}^{l}}{\operatorname{osc}} v .
$$

By (3.24) and (4.7), there exist constants $\theta \in(0,1)$ and $C>0$ such that

$$
\underset{E_{P_{0}}^{l-1}}{\operatorname{OSc} v \leq \theta}\left(1+C 2^{1+\frac{\epsilon^{\prime}\left(2-h_{0}+2 l\right)}{\kappa^{\prime}+\epsilon}}\right) \underset{E_{P_{0}}^{l}}{\operatorname{Oosc}} v
$$

for all $l \in\{1,2, \ldots, n\}$. By (4.8), we take a sufficiently large $h_{0}$ so that

$$
\underset{(x, t) \in E_{P_{0}}^{l-1}}{\operatorname{osc}} v(x, t) \leq \theta^{\prime} \underset{(x, t) \in E_{P_{0}}^{l}}{\operatorname{osc}} v(x, t), \quad l \in\{1,2, \ldots, n\}
$$

for some constant $\theta^{\prime} \in(\theta, 1)$. So we have

$$
\underset{E_{P_{0}}}{\operatorname{Osc}} v \leq \theta^{\prime n} \underset{E_{P_{0}}^{n}}{\operatorname{Oosc}} v
$$

Here we choose and fix $n$ so that $\theta^{\prime-n}>H^{10}$. Then by (4.9),

$$
\underset{E_{P_{0}}^{n}}{\operatorname{Osc}} v>2 H^{5\left(h_{0}+2\right)} v(\bar{P}) .
$$

Since $v$ is extended symmetrically across $x_{N}=0$, there exists a point $P_{1}=$ $\left(x_{1}^{\prime}, x_{1 N}, t_{1}\right) \in E_{P_{0}}^{n} \cap Q$ such that

$$
v\left(P_{1}\right)>H^{5\left(h_{0}+2\right)} v(\bar{P}) .
$$

Furthermore, by (4.6),

$$
0<x_{1 N}<2^{-h_{0}}
$$

Repeating this argument, we see that there exists a point $P_{2}=$ $\left(x_{2}^{\prime}, x_{2 N}, t_{2}\right)$ such that

$$
v\left(P_{2}\right)>H^{5\left(h_{0}+4\right)} v(\bar{P}), \quad 0<x_{1 N}<2^{-h_{0}-2} .
$$


By induction, we obtain a sequence $\left\{P_{m}\right\}$ such that

$$
v\left(P_{m}\right)>H^{5\left(h_{0}+2 m\right)} v(\bar{P}), \quad 0<x_{1 N}<2^{-h_{0}-2 m} .
$$

We choose a sufficiently large $h_{0}$ so that

$$
t_{0}-\sum_{m=1}^{\infty} 4^{2 n-h_{0}-2 m}>-\frac{3}{2}, \quad x_{0 i}-\sum_{m=1}^{\infty} 2^{2 n-h_{0}-2 m}>-3, i=1, \ldots, n-1 .
$$

Then the sequence $\left\{P_{m}\right\}$ is contained in a fix subcylinder of $\Psi$, and so this leads to a contradiction. Therefore, there exists a constant $C>0$ such that

$$
v(x, t) \leq C v(\bar{P}), \quad(x, t) \in \Psi^{\prime}
$$

and the proof of Theorem 4.1 is complete.

In the same way as in the proof of Theorem A, we see that (A4) implies (A4.c). Therefore, by Theorem 4.1, we have Theorem C.

Acknowledgements. The author wishes to thank Professor Minoru Murata for valuable advices.

\section{REFERENCES}

[CS1] F. Chiarenza and R. Serapinoi, Degenerate parabolic equations and Harnack inequality, Annali di Mat. Pura Appl., 4 (1983), 139-162.

[CS2] F. Chiarenza and R. Serapinoi, A Harnack inequality for degenerate parabolic equations, Comm. in P. D. E., 9 (1984), 719-749.

[CS3] F. Chiarenza and R. Serapinoi, A remark on a Harnack inequality for degenerate parabolic equations, Rend. Sem. Mat. Univ. Padova, 73 (1985), 179-190.

[CW] S. Chanillo and R. L. Wheeden, Harnack's inequality and mean-value inequalities for solutions of degenerate elliptic equations, Comm. in P. D. E., 11 (1986), 1111-1134.

[FGS] E. B. Fabes, N. Garofalo, and S. Salsa, A backward Harnack inequality and Fatou theorem for nonnegative solutions of parabolic equations, Illinois J. Math., 30 (1986), 536-565.

[FKS] E. B. Fabes, C. E. Kenig, and R. P. Serapioni, The local regularity of solutions of degenerate elliptic equations, Comm. P. D. E., 7 (1982), 77-116.

[GW1] C. E. Gutiérrez and R. L. Wheeden, Mean value and Harnack inequalities for degenerate parabolic equations, Colloquium Math., dedicated to A. Zygmund, LX/LXI (1990), 157-194. 
[GW2] C. E. Gutiérrez and R. L. Wheeden, Harnack's inequality for degenerate parabolic equations, Comm, P. D. E., 16 (1991), 745-770.

[HKO] J. Heinonen, T. Kilpeläinen, and O. Martio, Nonlinear potential theory of degenerate elliptic equations, Clarendon Press, New York, 1993.

[K] J. T. Kemper, A boundary Harnack principle for Lipschitz domain and the principle of positive singularities, Comm. Pure and Appl. Math., XXV (1972), 247-255..

[LSU] O. A. Ladyzeskayaya, N. S. Solonikov and N. N. Ural'tseva, Linear and quasilinear equations of parabolic type, Transl. Math. Monographs, 23, Amer. Math. Soc., Providence, R. I., 1968.

[Mr] C. B. Morrey, Multiple integrals in the calculus of variations, Springer-Verlag, Berlin-Heidelberg-New York, 1966.

[Ms] J. Moser, A Harnack inequality for parabolic differential equations, Comm. Pure Appl. Math., 17 (1964), 101-134.

[SaC1] L. Saloff-Coste, A note on Poincaré, Sobolev, and Harnack inequality, Duke Math. J., I. M. R. N., 2 (1992), 27-38.

[SaC2] L. Saloff-Coste, Parabolic Harnack inequality for divergence form second order differential operators, Potential Analysis, 4 (1995), 429-467.

[Sa] S. Salsa, Some properties of nonnegative solutions of parabolic differential operators, Ann. Mat. Pure Appl., 128 (1981), 193-206.

[Se] J. Serrin, Local behavior of solutions of quasi-linear equations, Acta Math., III (1964), 247-302.

[Sta] G. Stampacchia, Équations elliptiques du second ordre á coefficients discontinus, Les Presses De l'Université, 1966.

[Ste] E. M. Stein, Harmonic analysis, Princeton University Press, Princeton, 1992.

[T] N. S. Trudinger, Pointwise estimates and quasilinear parabolic equations, Comm. Pure Appl. Math., 21 (1968), 205-226.

Graduate School of Mathematics

Nagoya University

Chikusa-ku, Nagoya 464-8602

Japan

ishige@math. nagoya-u.ac.jp 